\title{
Associations between maternal psychological distress and salivary cortisol during pregnancy: A mixed-models approach
}

\author{
Marion I. van den Heuvel $^{\mathrm{a}}$, Marcel A.L.M. van Assen ${ }^{\mathrm{b}, \mathrm{c}}$, Vivette Glover ${ }^{\mathrm{d}}$, Stephan Claes ${ }^{\mathrm{e}}$, \\ Bea R.H. Van den Bergh ${ }^{f, g, *}$
}

${ }^{a}$ Department of Cognitive Neuropsychology, Tilburg University, The Netherlands

${ }^{\mathrm{b}}$ Department of Methodology and Statistics, Tilburg University, The Netherlands

${ }^{\mathrm{c}}$ Department of Sociology, Utrecht University, The Netherlands

${ }^{\mathrm{d}}$ Institute of Reproductive and Developmental Biology, Imperial College London, UK

e Department of Neurosciences, University of Leuven (KU Leuven), Leuven, Belgium

${ }^{\mathrm{f}}$ Health Psychology, University of Leuven (KU Leuven), Leuven, Belgium

${ }^{g}$ Department of Welfare, Public Health and Family, Flemish Government, Brussels, Belgium

\section{A R T I C L E I N F O}

\section{Keywords:}

Maternal cortisol

Fetal Programming

HPA-axis

Maternal depression

Maternal psychological distress

Maternal anxiety

\begin{abstract}
A B S T R A C T
Background: Maternal psychological distress during pregnancy is related to adverse child behavioral and emotional outcomes later in life, such as ADHD and anxiety/depression. The underlying mechanisms for this, however, are still largely unknown. The hypothalamic-pituitary-adrenal (HPA)-axis, with its most important effector hormone cortisol, has been proposed as a mechanism, but results have been inconsistent. The current study investigated the association between maternal psychological distress (i.e. anxiety and depressive symptoms) and maternal cortisol levels during pregnancy using a mixed models approach.

Method: During three pregnancy trimesters, mothers $(\mathrm{N}=170)$ collected four salivary samples for two consecutive days. Mothers reported symptoms of anxiety and depression three times during pregnancy (at $13.3 \pm 1.1,20.2 \pm 1.5$, and $33.8 \pm 1.5$ weeks of pregnancy, respectively) using the anxiety subscale of the Symptom Checklist (SCL-90), the Spielberger State and Trait Anxiety Inventory (STAI), and the Edinburgh Postnatal Depression Scale (EPDS). Specific fears and worries during pregnancy were measured with the short version of the Pregnancy Related Anxiety Questionnaire (PRAQ-R).

Results: We found a significant effect of SCL-90 anxiety subscale on cortisol levels at awakening $(\mathrm{p}=.008)$, indicating that mothers with higher anxiety showed lower cortisol at awakening. Maternal psychological variables explained $10.5 \%$ of the variance at the person level in awakening cortisol level, but none in the overall diurnal cortisol model.

Conclusion: More research is necessary to unravel the underlying mechanisms of the association between maternal psychological distress and cortisol and the search for mechanisms other than the HPA-axis should be continued and extended.
\end{abstract}

\section{Introduction}

Psychological distress during pregnancy is associated with a higher risk of behavioral and emotional problems, such as attention deficit hyperactivity disorder (ADHD), autism, and affective disorders in the offspring (e.g., Glover, 2014; Lahti et al., 2017; Van den Bergh and Marcoen, 2004; Van den Bergh et al., 2008, Van den Bergh et al., 2017, Walder et al., 2014). A frequently proposed mechanism for this association is the flow of abnormally high levels of maternal stress hormones, in particular cortisol, through the placenta (Beijers et al., 2014; Räikkönen et al., 2011). However, the evidence for an association between maternal psychological distress and cortisol levels during pregnancy has been inconsistent. While many studies report an association between maternal psychological state during pregnancy and maternal cortisol levels (Evans et al., 2008; Giesbrecht et al., 2012; Kalra et al., 2007; Kane et al., 2014; Kivlighan et al., 2008; O'Connor et al., 2014; Obel et al., 2005; Valsamakis et al., 2017), many other studies report non-significant findings (Baibazarova et al., 2013; Bleker et al., 2017; Hellgren et al., 2013; Himes and Simhan, 2011; Hompes et al., 2012; Petraglia et al., 2001; Salacz et al., 2012; Shea et al., 2007a; Voegtline et al., 2013). In addition, a recent systematic review by Zijlmans et al. (2015) investigating the association between maternal

\footnotetext{
* Corresponding author at: Health Psychology, Faculty of Psychology and Educational Sciences, University of Leuven (KU Leuven), Tiensestraat 102 - bus 3726, 3000 , Leuven, Belgium.

E-mail address: bea.vandenbergh@kuleuven.be (B.R.H. Van den Bergh).
} 
cortisol during pregnancy and child outcomes concluded that cortisol may not be the main underlying mechanism in the relation between maternal psychological distress and child outcomes.

Several methodological issues may play a role in the inconsistency of results. First of all, salivary cortisol can be measured in several different ways, such as using one or multiple samples. Because cortisol follows a typical pattern of secretion, with a cortisol awakening response (CAR) typically peaking around 30-40 minutes after awakening, and a diurnal profile with high levels in the morning and a decrease in cortisol levels through the end of the day (Edwards et al., 2001; Stalder et al., 2016), using a single sample of cortisol cannot capture all relevant information.

Another methodological issue is the use of different questionnaires. Many of the studies with non-significant results use general questionnaires of anxiety and depressive symptoms. Using pregnancy-specific questionnaires, asking about anxiety and depressive symptoms related to the pregnancy, could give a better view of stress the mothers are experiencing. Huizink et al. (2004) investigated whether pregnancy-related anxiety is distinct from general anxiety and concluded that pregnancy-related anxiety is a distinct syndrome and that it might be a better instrument to measure anxiety in pregnant women. Moreover, studies using pregnancy anxiety as predictor report significant association between maternal pregnancy anxiety and cortisol levels during pregnancy (e.g., Kane et al., 2014).

Most studies investigating the relation between maternal stress and cortisol secretion in pregnancy looked at the third trimester of pregnancy (e.g. Davis et al., 2007; Hellgren et al., 2013; Kivlighan et al., 2008; Simon et al., 2016). Maternal cortisol secretion changes over the course of the pregnancy, with 2-4-fold increases in cortisol from the first to the third trimester (de Weerth and Buitelaar, 2005; Sandman et al., 2006a). It is unclear whether the potential association between maternal stress and cortisol secretion is preserved throughout pregnancy, as some studies report attenuation with advancing gestation (Entringer et al., 2010), while others report no attenuation (Giesbrecht et al., 2013). By only looking at the third trimester, only a part of the association of maternal cortisol secretion during pregnancy and maternal psychological distress is determined. In addition, Obel et al. (2005) found that the association between psychological distress and cortisol level during pregnancy was dependent of the stage of pregnancy. More research is necessary to elucidate the changes of cortisol secretion over the course of pregnancy.

A further drawback of earlier studies is the use of statistical techniques that can only analyze time constant predictors, such as repeatedmeasures ANOVA. The amount of stress a mother experiences during her pregnancy, however, can change over the course of the pregnancy. We measured psychological distress in each trimester and used the more advanced mixed models that can incorporate both time varying and time constant variables (Heck et al., 2010; Hruschka et al., 2005). An important advantage of mixed models is the possibility of analyzing random effects, such as a random intercept (individual specific intercept) and a random slope (individual specific linear trajectory). In this way, individual differences in average cortisol level or in diurnal cortisol slope are taken into account (Hruschka et al., 2005). Since cortisol shows substantial within-subject variation (Giesbrecht et al., 2012), this is an important advantage. Moreover, mixed models are less sensitive to missing data, (a problem that frequently occurs in cortisol studies), than a repeated-measures technique. Repeated-measures ANOVA only analyses complete cases whereas mixed models use the available data more efficiently (Goldstein, 2003).

The aim of the current study was twofold: 1) replicating previous studies investigating maternal cortisol concentrations over the course of pregnancy, and 2) investigating the association between maternal selfreported psychological distress and cortisol concentrations during pregnancy. Based on previous literature, we expected to observe a cortisol awakening response throughout pregnancy and that maternal cortisol levels would increase in the course of pregnancy (de Weerth and Buitelaar, 2005; Sandman et al., 2006b). To overcome the abovementioned methodological issues we collected multiple salivary cortisol samples, measured psychological distress with both general questionnaires and a pregnancy-specific questionnaire, measured cortisol over the full course of pregnancy including the first trimester, and used a more advanced statistical technique (i.e., mixed models).

\section{Methods}

\subsection{Participants}

A total of 190 pregnant women were recruited during early to midpregnancy from four midwife practices and a general hospital between April 2009 and September 2010. All women participated in a longitudinal cohort following women, their partners and children from the first trimester of pregnancy onwards. All recruited cases included were singleton pregnancies, except for 1 case (included in the final sample). All participating mothers provided informed consent. The study was approved by the medical ethical committee of a local hospital, and was conducted in full compliance with the Helsinki declaration.

The final sample included 170 women. From the mothers enrolled in the study, 3 mothers did not participate in cortisol collection and from 2 mothers no cortisol could be detected in the collected samples. In addition, we excluded mothers suffering from conditions known to be associated with dysregulation of neuroendocrine function, such as hypothyroidism $(\mathrm{N}=10)$, and mothers who gave birth prematurely ( $<37$ weeks of gestation) $(\mathrm{N}=5)$. A total of 22 mothers dropped-out in the $2^{\text {nd }}$ or $3^{\text {rd }}$ trimester (i.e. no cortisol and no questionnaire data available). From these women, only data from the first (two) trimester (s) was used. Mothers dropped-out for several reasons: they did not want to participate in the study anymore $(\mathrm{N}=7)$, they did not send back the samples $(\mathrm{N}=5)$, a sick partner $(\mathrm{N}=1)$, sickness $(\mathrm{N}=1)$, and no reason given/not reachable $(\mathrm{N}=8)$. Additionally, 16 women were recruited in mid-pregnancy, after the collection of cortisol/questionnaires in trimester 1 was closed. For these women, only data from the second and/or third trimester(s) was used.

\subsection{Measurements}

All questionnaires were completed by the mothers three times during the pregnancy (i.e., for every trimester). We instructed the mothers to fill out the questionnaires digitally (web-based) one of the two days they collected the cortisol samples. A link to the questionnaires was send to mothers via email a few days before collection of cortisol. Mothers completed these questionnaires in their home setting.

\subsubsection{Anxiety}

Maternal self-reported state anxiety was measured in each trimester using the Dutch-version of the Spielberger State - Trait Anxiety Inventory (STAI; Van der Ploeg et al., 2000), containing 20 items scored from 1 to 4 , and the 10 -items anxiety subscale of the Symptom Checklist-90 (SCL-90) developed by Arrindel and Ettema (1981), also scored from 1 to 4 . Specific fears and worries during pregnancy were measured with the short Dutch-version of the Pregnancy Related Anxiety Questionnaire (Van den Bergh, 1990). The PRAQ-R consists of 5 subscales: concern for self ( 3 items), fear of integrity (4 items), fear of delivery ( 3 items), fear of changes ( 3 items) and concern for future relationships ( 4 items), with item scores ranging from 1 to 7 . According to a Dutch population-based study $(\mathrm{N}=6443)$, the cutoff for high state anxiety on the STAI is 43 (Koelewijn et al., 2017). Additionally, the cutoff for the SCL-90 anxiety subscale for "above average/high" for anxiety is 15 (Arrindell and Ettema, 2003). No norms are available for the PRAQ-R pregnancy-related anxiety scores.

\subsubsection{Depressive mood}

Maternal self-reported depressive symptoms were measured using 
the Edinburgh Postnatal Depression Scale (EPDS), a questionnaire developed by Cox et al. (1987) to screen mothers for depressive symptoms during pregnancy and the postnatal period. The questionnaire consists of 10 items scored from 0 to 3 about depressive symptoms of which a sum score can be calculated for overall depressive symptoms. According to Bergink et al. (2011), the clinical cutoffs of the Edinburgh Postnatal Depression Scale (EDS) based on a sample of pregnant women are 11 in the first trimester and 10 in the second and third trimester.

\subsubsection{Cortisol sampling}

Salivary cortisol was sampled over three trimesters of pregnancy. Four samples per day were taken for two consecutive days during the $8^{\text {th }}$ till the $14^{\text {th }}$ week of the pregnancy (first trimester), the $15^{\text {th }}$ till $22^{\text {nd }}$ week of the pregnancy (second trimester), and the $31^{\text {st }}$ till the $37^{\text {th }}$ week of the pregnancy (third trimester). We used specially designed test-tubes (Salivette ${ }^{\circledast}$, Sarstedt, Germany) to collect saliva. Mothers were instructed to take the first sample immediately after awakening, the second sample $30 \mathrm{~min}$ after awakening, the third $+6 \mathrm{~h}$ after the second, and the fourth $+6 \mathrm{~h}$ after the third. After taking the sample, the mothers were instructed to store the sample in a refrigerator and to return all collected samples by mail. In addition, they were instructed to write down the exact time of the sampling (i.e. hh:mm) and to report hours of sleep, the number of cigarettes smoked, sleep disturbance and working day/non-working day for each sampling day (cf. Stalder et al., 2016). After receiving, the collected samples were stored at $-20^{\circ} \mathrm{C}$ and shipped for analysis by Technische Universität Dresden (Kirschbaum, Dresden University of Technology, Germany). After thawing, saliva samples were centrifuged at 3000 for $5 \mathrm{~min}$. Salivary free cortisol was analyzed using chemiluminescence immunoassay (CLIA; IBL Hamburg, Germany). The precision of the intra- and inter-assay variability for the used technique is less than $10 \%$. The lower detection limit of this assay is $0.43 \mathrm{nmol}$ for a $50 \mu \mathrm{l}$ salivary sample. All samples of each subject were analyzed in the same run to reduce error variance caused by imprecision of the assay. Table 1 presents unadjusted cortisol values over the day (averaged over 2 days), separately for each trimester.

\subsubsection{Missing data}

For the cortisol samples, $25.3 \%$ was missing due to cortisol levels below detectable limits (13.5\%) and failure to collect/drop-out (11.8\%). For the time of sampling $15.0 \%$ was missing due to women forgetting to write down the time, forgetting the complete sample, or drop-out. Mixed models allow for the calculation of slopes and intercepts even with missing data and donot require an equal number of observations across individuals (Hruschka et al., 2005). The times that could be recalculated were manually imputed using the following rules: by one, two or three missing time(s) the time was recalculated with the other times of the day using our protocol (awaking, $+30 \mathrm{~min},+6 \mathrm{~h},+6 \mathrm{~h}$ ), by four missings on one day the times of the other day in the same trimester were copied, by missings on two days for one or two trimesters, the time of day of the other trimester was copied. The imputation resulted in a reduction of $4.1 \%$ of missing data on the time variable ( $10.9 \%$ missing data left). We ran the analysis on the data with and without imputed times and found no differences in interpretation of the results. If participants dropped-out ( $\mathrm{N}=22$; i.e., no cortisol, no questionnaires), data would still be included for the trimester(s) they did participate in. The majority of our

Table 1

Cortisol (nmol/l) per sample, per trimester (days aggregated).

\begin{tabular}{lllll}
\hline & Morning & Morning +30 min & $\begin{array}{l}\text { Noon } \\
(+6 \text { hours })\end{array}$ & $\begin{array}{l}\text { Afternoon } \\
(+6 \text { hours })\end{array}$ \\
\hline Trimester 1 & 20.63 & 24.98 & 9.33 & 4.82 \\
Trimester 2 & 22.61 & 27.62 & 10.68 & 6.09 \\
Trimester 3 & 24.20 & 30.73 & 14.68 & 9.21 \\
\hline
\end{tabular}

sample $(72.9 \%)$ contributes to all three trimesters, $18.2 \%$ contributes to 2 trimesters (either 1 and 3, 1 and 2, or 2 and 3), and only $8.8 \%$ of women only contributed to only 1 trimester. From some women we were unable to use the questionnaires (i.e., missing items, skipped full questionnaire): $9.4 \%, 11.2 \%$, and $18.2 \%$ for the first, second, and third trimester, respectively.

\subsection{Statistical analysis}

The cortisol samples were analyzed in two separate sets of analyses using mixed models, because previous research points to the CAR and the diurnal pattern as two separated entities, with weak correlations between the CAR and cortisol sampled over the day (Edwards et al., 2001). Moreover, twin studies documented a genetic influence on the CAR that is different from the heritability of daytime cortisol levels (Kupper et al., 2005). The first set (CAR-model) examined the influence of feelings of anxiety and depressed mood on cortisol at awakening, modeled by the intercept of the CAR-model, and the CAR, modeled by the slope, i.e. the change in cortisol from the first to second measurement per hour. The second set (DIURNAL-model) examined the effect of these influences on the diurnal cortisol profile including the first, third and fourth cortisol salivary samples.

For both sets the logarithm of cortisol was taken as the dependent variable, since the distribution of cortisol was found to be skewed (cf. Giesbrecht et al., 2012; Harville et al., 2007; Hruschka et al., 2005). Taking the logarithm of cortisol normalized the distribution of our data. Each of the cortisol measurements was averaged over the two measurement days within the same trimester. For a better interpretation of coefficients of the psychological variables those variables were grand mean centered (i.e. the overall mean of a variable was subtracted from all scores on that variable). Time of sampling was centered at awakening (i.e. the first sample was the reference and was set to zero).

Different models were estimated and compared to find the model that best fitted the data guided by the proposed steps of Hruschka et al. (2005). We started with a full-factorial model only including time of sampling and trimester (both within-person and time-varying variables), with a random intercept. Time of sampling corresponds to the exact time (hh:mm) the mother took the salivary sample. Trimester was defined as first, second, and third measurement period (coded 1, 2, or 3 ), which corresponds to the pregnancy trimester, rather than the exact gestational weeks. The DIURNAL-model also included a quadratic effect of time (of sampling), to investigate whether the steepness of the diurnal rhythm changed over the course of pregnancy and whether it is related to maternal psychological functioning. We proceeded with a model only including effects with $p<.10$. This model was then extended with a random effect of time, and the covariance between this effect and the random intercept, which allows for individual differences in the effect of time on the cortisol level (Snijders and Bosker, 2011). With a Deviance-test $(\alpha=.05)$ using restricted maximum likelihood (REML) we tested whether extending the model with a random slope explained significant extra variation in cortisol. REML was also used to calculate the variances explained by the psychological variables. The model that fitted the data best ("basic model") was then used to examine whether cortisol at awakening, the CAR, and the diurnal cortisol level changed over pregnancy (hypothesis 1). For interpretation of the fixed effects, we used and reported the models fitted with maximal likelihood (ML). The Aikake Information Criterion (AIC) was used for model selection.

To examine the association between maternal psychological functioning and maternal cortisol level (hypothesis 2), we extended the basic model with psychological variables (i.e., maternal self-reported anxiety and depressive symptoms). Additionally, we added the following potential confounders to the model: type of day (weekend or weekday), sleep disturbance, hours of sleep, time of waking, maternal BMI (at recruitment), and maternal parity. Only maternal BMI and parity were time-invariant. Time of waking was added in addition to 
time of sampling (i.e., time of acquiring salivary sample), to control for the time the participants woke up. Next, interactions with time of sampling and psychological variables were tested in the model. Again, we proceeded with a model that only included effects with $p<.10$. We examined whether the selected psychological variables significantly improved the fit of the model using a Deviance-test with ML, testing the basic model against the basic model including the selected psychological variables.

For the ease of interpretation we also reported the significant coefficients in terms of $\%$ change per SD [formula: $((\exp (\beta * \mathrm{SD})-1) * 100]$ in line with the work of Kivlighan et al. (2008) and Giesbrecht et al. (2012). For the psychological variables, the standard deviation over all three trimesters was used. The formula reverses the LN-transformation so that cortisol levels are presented in the original scale, nmol/l. We also computed how much variance was explained by the psychological variables in total, and at the residual, person, and trimester level, for onset in the CAR model and $6 \mathrm{~h}$ after the first measurement for the diurnal model (Raudenbush and Bryk, 2002). Explained variances can be smaller than 0 in mixed models, which cannot occur in regression analysis. Negative variance can be interpreted as no to low explained variance.

\section{Results}

\subsection{Descriptives}

The mothers had a mean age of 34.0 (SD $=4.9)$ in the first trimester. At the time of measurement, the women were on average $13.3 \pm 1.1,20.2 \pm 1.5$, and $33.8 \pm 1.5$ weeks pregnant in the first, second and third trimesters, respectively. Regarding depression scores on the EPDS, $9.3-18.4 \%$ of the women in our sample classified as "depressed" during pregnancy, with highest percentages in the third trimester (18.4\%). For anxiety, rates of high state anxiety (STAI questionnaire) in early, mid- and late pregnancy (12.6-16.5\%) were relatively low as compared to the rates of high state anxiety (30.9\%) during the first half of pregnancy in a recent Dutch population-based study ( $\mathrm{N}=6443$ ) (Koelewijn et al., 2017). Additionally, between 18.4-26.5\% of women reported scores classified as "above average/high" for anxiety according to the SCL-90 subscale norms (Arrindell and Ettema, 2003). More information about characteristics of the sample, including age, race/ethnicity, education, parity, and BMI can be found in Supplemental Table S1.

The number of participants, mean, standard deviation, and Cronbach's alpha of each psychological stress variable are presented in Table 2, for each trimester separately. The Cronbach's alpha's of the questionnaires were around .80, with the exception of the concern for future relationships subscale of the PRAQ-R which had a reliability closer to .70. Inter-correlations of the psychological stress variables ranged from 0.011 to 0.756 , with the majority of correlations between .2 and .4. See Supplementary Table S2 for correlations between the psychological variable, separately per trimester. Additionally, collinearity statistics were all acceptable (VIF $<10$; Tolerance $<0.2$; Menard, 1995; Myers, 1990). Finally, we confirmed that about $>70 \%$ of our included mothers showed a positive cortisol awakening response (versus a flat or declining slope).

\subsection{Drop-out analyses}

Possible drop-out bias was examined by testing differences between the drop-outs and non-drop-outs with two-sample $t$-tests using a corrected alpha-level corrected for multiple testing with Bonferroni correction $[\alpha=\alpha / n=.05 / 9=.006$, with $n$ as number of tests] for cortisol levels and scores on the psychological variables in the first trimester. After controlling for multiple testing, the drop-outs did not have higher cortisol levels in the first trimester than the non-drop-outs, nor significantly higher scores on the psychological variables. We note that the
Table 2

Descriptive statistics of psychological variables.

\begin{tabular}{|c|c|c|c|c|}
\hline Variable & Mean & Range & SD & $\alpha$ \\
\hline \multicolumn{5}{|l|}{ Trimester 1} \\
\hline EPDS & 5.78 & $0-17$ & 4.30 & .811 \\
\hline SCL-90 anxiety & 14.53 & $10-36$ & 5.20 & .874 \\
\hline STATE anxiety & 34.86 & $23-63$ & 8.01 & .927 \\
\hline PRAQ-R - concern for self & 2.61 & $1-6.33$ & 1.14 & .774 \\
\hline PRAQ-R - fear of integrity & 2.99 & $1-6.50$ & 1.48 & .868 \\
\hline PRAQ-R - fear of delivery & 2.59 & $1-6.33$ & 1.41 & .802 \\
\hline PRAQ-R - fear of changes & 2.56 & $1-7$ & 1.42 & .765 \\
\hline PRAQ-R - concern future relationships & 1.99 & $1-4.5$ & 0.97 & .710 \\
\hline \multicolumn{5}{|l|}{ Trimester 2} \\
\hline EPDS & 4.46 & $0-20$ & 4.09 & .837 \\
\hline SCL-90 anxiety & 13.67 & $10-38$ & 4.40 & .849 \\
\hline STATE anxiety & 33.65 & $23-65$ & 7.18 & .914 \\
\hline PRAQ-R - concern for self & 2.44 & $1-6$ & 1.04 & .749 \\
\hline PRAQ-R - fear of integrity & 2.71 & $1-7$ & 1.53 & .909 \\
\hline PRAQ-R - fear of delivery & 2.60 & $1-6.67$ & 1.46 & .793 \\
\hline PRAQ-R - fear of changes & 2.43 & $1-6.33$ & 1.42 & .752 \\
\hline PRAQ-R - concern future relationships & 1.90 & $1-5.5$ & 0.98 & .748 \\
\hline \multicolumn{5}{|l|}{ Trimester 3} \\
\hline EPDS & 5.34 & $0-21$ & 4.64 & .857 \\
\hline SCL-90 anxiety & 13.33 & $10-32$ & 3.71 & .808 \\
\hline STATE anxiety & 35.25 & $23-68$ & 7.90 & .924 \\
\hline PRAQ-R - concern for self & 2.49 & $1-5.67$ & 1.07 & .802 \\
\hline PRAQ-R - fear of integrity & 2.61 & $1-7$ & 1.46 & .898 \\
\hline PRAQ-R - fear of delivery & 2.78 & $1-6.67$ & 1.34 & .780 \\
\hline PRAQ-R - fear of changes & 2.33 & $1-5.67$ & 1.30 & .789 \\
\hline PRAQ-R - concern future relationships & 1.94 & $1-5.25$ & 0.92 & .719 \\
\hline
\end{tabular}

Notes: EPDS $=$ Edinburgh Depression Scale; SCL-90 $=$ Symptom Check List 90, STATE $=$ Spielberger State and Trait Anxiety Inventory; PRAQ-R = Pregnancy Related Anxiety Questionnaire; SD = standard deviation; $\alpha=$ Cronbach's Alpha.

uncorrected test statistics suggest that the drop-outs had higher fear of changes (PRAQ questionnaire; $\mathrm{t}=-2.588, \mathrm{p}=0.011$ ) and lower morning cortisol $(\mathrm{t}=2.083, \mathrm{p}=0.039)$.

\subsection{CAR-model}

For the CAR-model, the interaction of trimester and time of measurement was not significant (i.e., the CAR was not affected by pregnancy trimester), and therefore only the main effects of trimester and time were selected for the final model. The addition of a random effect of time significantly improved the model $\left[\chi^{2}(\mathrm{df}=2)=7.304\right.$; $p=.026]$, indicating that there were between-individual differences in CAR (Hruschka et al., 2005). The final 'basic model' included fixed effects of trimester, random intercept, and random effects of time and trimester (Table 3).

The fixed-effects coefficients of the final basic model of the CARmodel showed a positive CAR $(p<.001$; coefficient indicated an increase of $19.7 \%$ per half hour). Women on average experienced $21.7 \%$ higher levels of cortisol in the third trimester compared to the first trimester $(p<.001)$, and $8.0 \%$ higher cortisol compared to the second trimester $(p=.015)$.

The psychological predictors and their interactions with time were added to the basic CAR-model next. Selecting on the basis of $p=.10$ yielded a model including the following psychological predictors: SCL90 anxiety, fear of changes PRAQ-subscale, and their interactions with time. Next, we tested whether these four psychological predictors predicted cortisol levels, using Bonferroni correction for multiple testing $[\alpha=.05 / 4=.0125]$. Adding the four psychological predictors to the basic model improved the fit of the model $\left[\chi^{2}(4)=14.574 ; p=.006\right]$. However, only the main effect of SCL-90 anxiety was significant $(p=.008)$, indicating lower awakening cortisol levels (i.e., lower intercept) were associated with higher anxiety as measured with the SCL90 anxiety subscale $(5.2 \%$ per SD; see Fig. 1$)$. Note that the results are not affected by the Bonferroni corrections, as the $p$-values of the tests of 
Table 3

Final basic CAR-model with coefficients \& CAR-model with psychological predictors.

\begin{tabular}{|c|c|c|c|c|}
\hline \multicolumn{5}{|c|}{ Basic CAR-Model } \\
\hline Fixed effects & Coefficient & S.E. & t-statistic & Interpretation \\
\hline Intercept & 3.168 & .031 & $102.754^{* * * *}$ & $\begin{array}{l}\text { Grand average cortisol on } \mathrm{T}=0 \text {, } \\
\text { Trim }=3\end{array}$ \\
\hline Trimester $=1$ & -0.245 & .035 & $-7.075^{* k \ldots k}$ & $\begin{array}{l}-21.7 \% \text { less cortisol than in } \\
\text { Trim }=3\end{array}$ \\
\hline Trimester $=2$ & -0.085 & .035 & $-2.454^{*}$ & $\begin{array}{l}-8.1 \% \text { less cortisol than in } \\
\text { Trim }=3\end{array}$ \\
\hline Time & 0.332 & .038 & $8.846^{\text {**** }}$ & $19.7 \%$ increase per half hour \\
\hline
\end{tabular}

\begin{tabular}{llll}
\hline Random effects $\perp$ & Variance component & S.E. & Wald z \\
\hline Residual & 0.061 & 0.0058 & $10.567^{\text {***** }}$ \\
Intercept (person-level) & 0.038 & 0.010 & $3.674^{\text {**** }}$ \\
Covariance (person-level) & -0.017 & 0.012 & -1.40 \\
Time (person-level) & 0.052 & 0.024 & $2.191^{*}$ \\
Intercept (trimester-level) & 0.039 & 0.0079 & $4.889^{\text {***** }}$ \\
\hline
\end{tabular}

CAR-model with psychological predictors

\begin{tabular}{|c|c|c|c|c|c|}
\hline Fixed effects & Coefficient & S.E. & t-statistic & \multicolumn{2}{|c|}{ Interpretation } \\
\hline Intercept & 3.168 & .030 & $104.753^{* * * k x}$ & \multicolumn{2}{|c|}{$\begin{array}{l}\text { Grand average cortisol on } \\
\mathrm{T}=0 \text {, Trim }=3\end{array}$} \\
\hline Trimester $=1$ & -.232 & .035 & $-6.653^{3.1 \times k x}$ & \multicolumn{2}{|c|}{$\begin{array}{l}-20.7 \% \text { less cortisol than } \\
\text { in Trim }=3\end{array}$} \\
\hline Trimester $=2$ & -.083 & .034 & $-2.42^{*}$ & \multicolumn{2}{|c|}{$\begin{array}{l}-8.0 \% \text { less cortisol than in } \\
\text { Trim }=3\end{array}$} \\
\hline SCL-90 anxiety & -.012 & .0045 & $-2.681^{* *}$ & \multicolumn{2}{|c|}{$-5.2 \%$ decrease per $\mathrm{SD}^{\mathrm{a}}$} \\
\hline $\begin{array}{l}\text { PRAQ: Fear of } \\
\text { changes }\end{array}$ & -.027 & .015 & -1.755 & \multicolumn{2}{|l|}{ n.s. } \\
\hline Time & .328 & .037 & $8.799^{* \cdots \cdots k}$ & \multicolumn{2}{|c|}{$\begin{array}{l}19.4 \% \text { increase per half } \\
\text { hour }\end{array}$} \\
\hline SCL-90 anxiety & .012 & .0084 & 1.430 & \multicolumn{2}{|l|}{ n.s. } \\
\hline $\begin{array}{l}\text { PRAQ: Fear of } \\
\text { changes }\end{array}$ & -.052 & .028 & 1.874 & \multicolumn{2}{|l|}{ n.s. } \\
\hline Random effects $\perp$ & & \multicolumn{2}{|c|}{ Variance component } & S.E. & z-statistic \\
\hline Residual & & \multicolumn{2}{|l|}{.060} & .0057 & $10.519^{* * * * *}$ \\
\hline Intercept (person-level) & & \multicolumn{2}{|l|}{.034} & .010 & $3.398^{* * *}$ \\
\hline Covariance (person-lev & vel) & \multicolumn{2}{|l|}{-.014} & .012 & -1.190 \\
\hline Time (person-level) & & \multicolumn{2}{|l|}{.055} & .025 & $2.229^{*}$ \\
\hline Intercept (trimester-lev & vel) & \multicolumn{2}{|l|}{.040} & .0078 & $4.990^{* * \ldots \times k}$ \\
\hline
\end{tabular}

$* p<.05$.

$* * p<.01$.

$* * * p<.001$

a SD of SCL-90 anxiety questionnaire $=4.44$; SCL-90 $=$ Symptom Checklist 90; PRAQ = Pregnancy Related Anxiety Questionnaire. Intercept refers to the third trimester (reference point). Model coefficients reported are uncorrected for confounders. $\perp$ Random effects estimates are shown of the REML model.

the three other psychological predictors exceed .05. Finally, we tested whether the results remained significant after controlling for confounders. None of the confounders were significantly associated with cortisol concentrations in the CAR-model and controlling for confounders did not change the above reported results. The uncorrected model (without confounders) and all its coefficients are represented in Table 3.

The random effects indicate that the first cortisol measurement varied across persons and trimesters, whereas the CAR varied across persons as well. Comparing the random effect variances, the psychological characteristics explained $2.9 \%$ of the total variance, and $1.6 \%$, $10.5 \%,-2.6 \%$ at the residual, person, trimester level, respectively. While the psychological variables explained little of mother's CAR in total and at the trimester level, a considerable amount of variance, i.e., $10.5 \%$, is explained by the psychological variables at the person level. This indicates that individual differences in CAR (person level), but not differences in CAR as result of trimester (trimester level), are explained by psychological distress.

\subsection{DIURNAL-model}

For the DIURNAL-model, the fixed effects of time (linear and quadratic), trimester, and the interaction of time (only linear) and trimester were included in the final basic model. The addition of a random effect of time significantly improved the model $\left[\chi^{2}(2)=39.468\right.$; $p<.001]$, indicating that profiles differ between individuals. The final basic model and its coefficients are presented in Table 4.

The results of the final basic DIURNAL-model showed a main effect of trimester on cortisol levels with $24.3 \%$ higher levels on trimester 3 than trimester $1(p<.001)$, as was found in the CAR-model as well. There is no difference in cortisol levels found between trimester 2 and 3 $(p=.104)$. Time of the measurement was also significant $(p<.001)$, with a coefficient indicating a $4.3 \%$ decline of cortisol level every half hour. The coefficient of the quadratic effect of time indicates that this decline flattens over time with $0.1 \%$ per half hour. In other words, the slope of the decline in cortisol level that we observed over the day in pregnant women becomes less steep over time of day. The interaction between time of day and trimester is also significant $(p=.018)$. The results showed a significant flatter slope for trimester 3 compared to trimester 1 and 2, suggesting less decline in cortisol levels over the day, possibly resulting in a higher evening cortisol level, for mothers in late pregnancy.

The psychological predictors and their interactions with time were added to the basic DIURNAL model next. Selecting on the basis of $p=.10$ yielded a model including the following psychological predictors: SCL-90 anxiety, fear of changes PRAQ-subscale, and the interaction between fear of changes PRAQ-subscale and time. Adding these three psychological predictors improved the fit of the model $\left[\chi^{2}\right.$ (3) $=8.037 ; p=.045]$. Next, we tested whether these psychological predictors predicted cortisol levels, using Bonferroni correction for multiple testing $[\alpha=.05 / 3=.0166$, with three psychological predictors]. We found a significant interaction effect between the fear of changes PRAQ-subscale and time $(\mathrm{p}=0.018)$, indicating that a flatter diurnal decline was associated with higher levels of pregnancy-specific anxiety as measured with the fear of changes subscale of the PRAQ-R. Nevertheless, this association was small and disappeared after correcting for multiple testing as well as after controlling for confounders. None of the confounders were significantly associated with cortisol concentrations in the DIRUNAL model. The uncorrected model (without confounders) and its coefficients are represented in Table 4.

The random effects indicate that the first cortisol measurement and the linear effect of time on cortisol varied across persons, and cortisol levels varied across waves as well. The psychological variables did not explain total variance $(-1.7 \%)$, and no variance at the residual $(0 \%)$, person $(-2.3 \%)$, and wave level $(-2.8 \%)$ either. We therefore conclude that the effects of psychological distress on diurnal cortisol levels of mothers during pregnancy are weak at best.

\section{Discussion}

The aim of the current study was to examine (1) how the CAR and the diurnal cortisol level changes over pregnancy, from first to third trimester, and (2) whether maternal self-reported anxiety and depressive mood are associated with maternal cortisol in pregnant women. The findings of our study contribute to the previous knowledge regarding cortisol response during pregnancy and the effect of psychological distress on these levels. Previous studies on the effect of maternal psychological distress during pregnancy and cortisol levels have yielded inconsistent results and lacked more powerful mixed modeling analyses. This study addresses that gap in our knowledge, by reporting effect sizes and interpretations of the contribution of each maternal psychological factor. Overall, our results suggest that maternal psychological distress during pregnancy and salivary cortisol levels are 


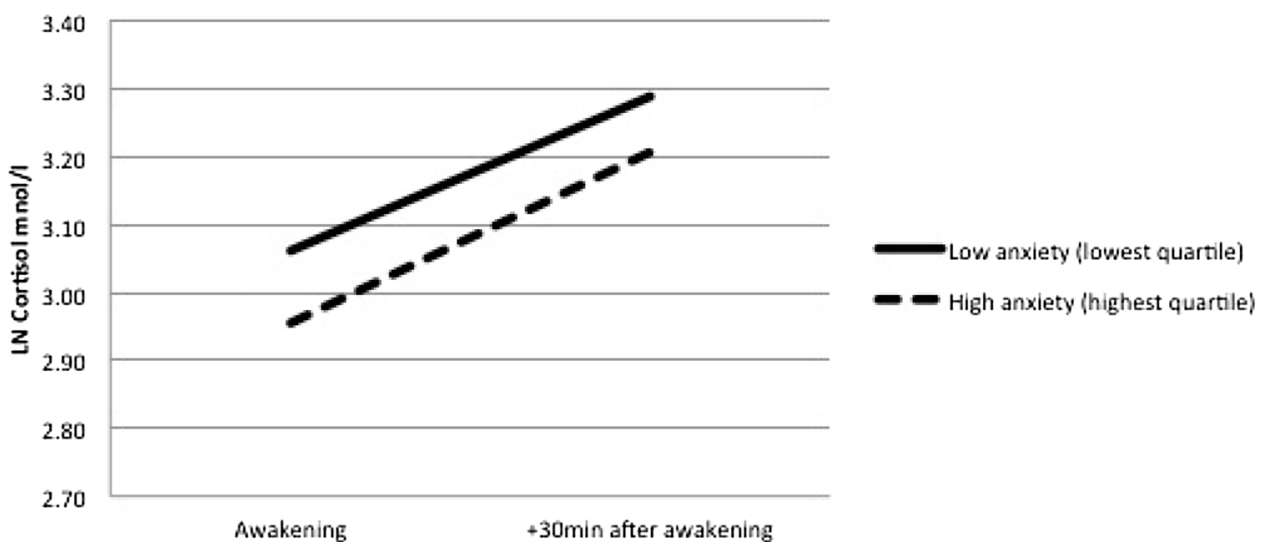

Fig. 1. Graphical representation of the effect of maternal anxiety (SCL-90) on awakening cortisol levels in the CAR-model. Cortisol levels are plotted for mothers with low anxiety (lowest quartile; cut-off $=11$; solid line) and high anxiety (highest quartile; cut-off $=15$; dashed line). Means are averaged over trimesters.

weakly related at best.

\subsection{Maternal cortisol over the course of pregnancy}

In line with our expectations and previous studies (de Weerth and Buitelaar, 2005; Sandman et al., 2006b), mothers in our study displayed the typical CAR and diurnal pattern of cortisol secretion in each trimester of pregnancy, with increasing cortisol levels over the course of pregnancy. In line with earlier studies in a pregnant sample (e.g., Kivlighan et al., 2008), we demonstrated that pregnant women display a significant diurnal pattern showing a decline from morning to evening. Additionally, the significant quadratic effect of time showed that the decline from morning to evening flattened over the day. By testing interactions between the diurnal slope and trimester, we also showed a significant flatter decline of the diurnal profile in the third trimester compared to the first and the second.

\subsection{Associations between maternal psychological functioning and cortisol during pregnancy}

We found a small negative association between the SCL-90 anxiety subscale and the CAR-model intercept, indicating that mothers with higher anxiety show lower awakening cortisol levels. In other words, our data suggest that more anxious mothers start the day with lower cortisol values than those with lower anxiety. Importantly, deviance tests revealed that adding the psychological measures to the model significantly improved model fit and we found that $10.5 \%$ of personlevel variance was explained by the psychological variables. The result of lower awakening cortisol in pregnant women experiencing higher anxiety is in line with other studies that reported a lower morning cortisol levels and decreased CAR in depressed pregnant women (Simon et al., 2016) and distressed Black pregnant women (Suglia et al., 2010). However, there are also studies that reported no association between the CAR and psychological distress during pregnancy (Hellgren et al., 2013; Shea et al., 2007b). In the non-pregnant population, lower morning cortisol has been associated with burnout, fatigue, exhaustion, and sleep problems (Backhaus et al., 2004; Chida and Steptoe, 2009). For the diurnal cortisol profile, no significant associations were found. Additionally, the overall deviance test of psychological predictors was not statistically significant for diurnal cortisol levels.

The fact that we only found statistically significant associations for the SCL-90 anxiety subscale and not for any of the other anxiety subscales could be because this measure focuses heavily on somatic symptoms of anxiety, whereas the other anxiety questionnaires focus more on the psychological symptoms related to anxiety (Bech, 2011). Somatic anxiety symptoms, such as trembling and feeling restless, may be stronger related to having altered cortisol levels than psychological symptoms, such as worrying and fearful thoughts. However, we cannot exclude that the different patterns of results are mainly caused by sampling error.

Based on the results of the deviance test and explained variances, we conclude that, in a community sample of pregnant women, psychological distress variables may play a small role in awakening cortisol levels, but likely do not affect the diurnal cortisol levels. Many previous studies reported no association between psychological functioning and maternal cortisol during pregnancy in community samples (Baibazarova et al., 2013; Hellgren et al., 2013; Himes and Simhan, 2011; Hompes et al., 2012; Petraglia et al., 2001; Salacz et al., 2012; Shea et al., 2007a; Voegtline et al., 2013). Studies that found significant result reported small effects and concluded that, in general, maternal psychological distress had little impact on the cortisol levels of the pregnant women in their study (Kivlighan et al., 2008; O'Connor et al., 2014).

\subsection{Strengths and limitations}

Our study has some advantages over other studies. To the best of our knowledge, this is the first study using mixed modeling, which allows for distinguishing between explained variance within and between persons, may include time-varying predictors, and can deal with missing values of the dependent variable. Moreover, the multilevel technique used in this study provides valuable extra information on cortisol secretion over the course of pregnancy, since this study incorporates trimester as an extra level into a multilevel model, which enables statistical testing of hypotheses that are often only described or not longitudinally tested. Other strengths of the current study include the collection of multiple samples over multiple days, the inclusion of a relatively large group of pregnant women, and the use of pregnancyrelated anxiety questionnaires. These strengths, together with advanced statistical modelling, could all have contributed to higher power in finding effects.

Some limitations of the study should also be mentioned. First of all, the measurement of anxiety and depressive symptoms relies on selfreport. This leaves room for recall-bias as well as socially desirable answering. Related to this, although our study measured cortisol and maternal psychological distress on the same day, it was not measured at the same time of the day and only one measure of distress per day was taken. This measurement protocol is prone to numerous systematic biases, including availability heuristics (i.e., the influence of emotional states and arousal at the time of encoding and recall affect memories). Ecological momentary measurement (EMA) could be used in future studies to enable simultaneous measurement of cortisol and mood (e.g., Giesbrecht et al., 2013, Giesbrecht et al., 2012). Although EMA is believed to reveal stronger associations than general self-report 
Table 4

Final basic DIURNAL-model with coefficients \& DIURNAL-model with psychological predictors.

\begin{tabular}{|c|c|c|c|c|c|}
\hline \multicolumn{6}{|c|}{ Basic DIURNAL-model } \\
\hline Fixed effects & Coefficient & S.E. & t-statistic & \multicolumn{2}{|l|}{ Interpretation } \\
\hline Intercept & 3.169 & .034 & $92.381^{* k * * *}$ & \multirow{2}{*}{\multicolumn{2}{|c|}{$\begin{array}{l}\text { Grand average cortisol on } \\
\mathrm{T}=0 \text {, Trim }=3 \\
-24.3 \% \text { less cortisol than in } \\
\text { Trim }=3\end{array}$}} \\
\hline Trimester $=1$ & -.278 & .043 & $-6.453^{\text {k.kk }}$ & & \\
\hline Trimester $=2$ & -.071 & .043 & -1.626 & \multirow{5}{*}{\multicolumn{2}{|c|}{$\begin{array}{l}\text { n.s. } \\
-4.3 \% \text { decline per half hour } \\
.1 \% \text { flatter per half hour } \\
-2.1 \% \text { more decline per half } \\
\text { hour than in Trim }=3 \\
-1.9 \% \text { more decline per half } \\
\text { hour than in Trim }=3\end{array}$}} \\
\hline Time & -.091 & .0070 & $-13.064^{* * * *}$ & & \\
\hline Time & .0011 & .00048 & $2.251^{*}$ & & \\
\hline Trimester $=1$ & -.042 & .0045 & $-9.250^{\text {v.k.kn}}$ & & \\
\hline Trimester $=2$ & -.038 & .0045 & $-8.314^{k * k k k}$ & & \\
\hline \multicolumn{2}{|c|}{ Random effects $\perp$} & \multicolumn{2}{|c|}{ Variance component } & S.E. & z-statistic \\
\hline \multicolumn{2}{|l|}{ Residual } & \multicolumn{2}{|l|}{.089} & .0053 & $16.858^{k * * s}$ \\
\hline \multicolumn{2}{|c|}{ Intercept (person-level) } & \multicolumn{2}{|l|}{.025} & .0086 & $2.903^{* *}$ \\
\hline \multicolumn{2}{|l|}{ Covariance } & \multicolumn{2}{|c|}{-.0016} & .00079 & $-1.979^{*}$ \\
\hline \multicolumn{2}{|c|}{ Slope (person-level) } & \multicolumn{2}{|c|}{.00047} & .00011 & $4.104^{* * * x \cdot}$ \\
\hline \multicolumn{2}{|c|}{ Intercept (trimester-level) } & \multicolumn{2}{|c|}{.036} & .0069 & $5.338^{* \ldots * k}$ \\
\hline
\end{tabular}

DIURNAL-model with psychological predictors

\begin{tabular}{|c|c|c|c|c|c|}
\hline Fixed effects & Coefficient & S.E. & t-statistic & \multicolumn{2}{|c|}{ Interpretation } \\
\hline Intercept & 3.166 & .034 & $93.199^{* * * *}$ & \multicolumn{2}{|c|}{$\begin{array}{l}\text { Grand average cortisol } \\
\text { on } \mathrm{T}=0, \text { Trim }=3\end{array}$} \\
\hline Trimester $=1$ & -.266 & .043 & $-6.173^{\text {nitke }}$ & \multicolumn{2}{|c|}{$\begin{array}{l}-41.0 \% \text { less cortisol } \\
\text { than in Trim }=3\end{array}$} \\
\hline Trimester $=2$ & -.069 & .043 & -1.586 & \multicolumn{2}{|c|}{ n.s. } \\
\hline SCL-90 anxiety & -0.0054 & 0.0036 & -1.499 & \multicolumn{2}{|l|}{ n.s. } \\
\hline $\begin{array}{l}\text { PRAQ: Fear of } \\
\text { changes }\end{array}$ & -.021 & 0.015 & -1.399 & \multicolumn{2}{|l|}{ n.s. } \\
\hline Time & -0.091 & 0.0070 & $-13.085^{* * * k}$ & \multicolumn{2}{|c|}{$\begin{array}{l}-5.7 \% \text { decrease per half } \\
\text { hour }\end{array}$} \\
\hline Time & 0.001 & 0.001 & $2.283^{*}$ & \multirow{2}{*}{\multicolumn{2}{|c|}{$\begin{array}{l}0.1 \% \text { flatter per half hour } \\
-2.08 \% \text { more decline } \\
\text { than in Trim }=3\end{array}$}} \\
\hline Trimester $=1$ & -.043 & .0045 & $-9.484^{* * * k}$ & & \\
\hline Trimester $=2$ & -.038 & .0045 & $-8.545^{n+k+k}$ & \multicolumn{2}{|c|}{$\begin{array}{l}-1.86 \% \text { more decline } \\
\text { than in Trim }=3\end{array}$} \\
\hline $\begin{array}{c}\text { PRAQ: Fear of } \\
\text { changes }\end{array}$ & 0.0040 & 0.0017 & $2.372^{*, \mathrm{a}}$ & $.37 \%$ flat & er $\mathrm{SD}^{\mathrm{b}}$ \\
\hline Random effects $\perp$ & & \multicolumn{2}{|c|}{ Variance component } & S.E. & z-statistic \\
\hline Residual & & .089 & & .0053 & $16.847^{* * * *}$ \\
\hline Intercept (person-leve & & .023 & & .0083 & $2.777^{*}$ \\
\hline Covariance & & -.0014 & & .00079 & -1.806 \\
\hline Slope (person-level) & & .00048 & & .00012 & $4.162^{* * \times 1 \times k}$ \\
\hline Intercept (trimester-le & evel) & .037 & & .0069 & $5.333^{\text {*ntkex }}$ \\
\hline
\end{tabular}

$* p<.05$.

$* * p<.01$.

$* * * p<.001$.

a No longer significant after controlling for confounders; ${ }^{b}$ SD for PRAQ: Fear of changes $=1.38$; SCL-90 $=$ Symptom Checklist 90; PRAQ $=$ Pregnancy Related Anxiety. Model coefficients reported are uncorrected for confounders. $\perp$ Random effects estimates are shown of the REML model.

questionnaires, there are not many studies that used these methods in samples of pregnant women yet. Therefore, it is uncertain how much stronger an association these methods would reveal. Second, though the investigators developed a clear protocol and a comprehensive instruction letter for the collection of the samples, we cannot state with certainty that all samples were taken as was intended and/or as reported. All mothers were very dedicated to the sample collection and we confirmed that most included mothers (70-78\%) showed a positive CAR. While a high percentage of positive CARs is only a proxy measure of compliance and flat CARs can be observed even in compliant women, the high percentage suggests compliance to our collection protocol.
Nevertheless, it is possible that they made errors with writing down the times of sampling, hours of sleep, etc. Another limitation is the fact that the mothers included in the current study did not display large variation in psychological distress level. There are few mothers who reported clinical levels of distress. However, studies examining maternal cortisol in pregnant women diagnosed with depressive disorder also reported no relation between maternal cortisol levels and depression (Hellgren et al., 2013; Salacz et al., 2012). Moreover, we and other groups have found associations between maternal distress during pregnancy and child outcomes in similar samples of women (e.g., O'Connor et al., 2002; Van den Bergh and Marcoen, 2004; van den Heuvel et al., 2015; Zijlmans et al., 2017). Finally, due to financial constraints we used four salivary cortisol samples, but more are always better. Especially for measuring the CAR, more samples in the first hour after awakening raise the reliability of the results.

\section{Conclusion}

The results of the current study show that maternal self-reported anxiety and salivary cortisol levels in a community sample of pregnant women are at best weakly associated. Deviance tests and random effects, suggest only small contributions of maternal psychological distress during pregnancy in explaining the variation in cortisol awakening level, and no contribution in diurnal cortisol levels. Due to the use of a more advanced statistical methods and a relatively large amount of data (i.e., multiple samples over two days in each pregnancy trimester in a relatively large group of pregnant women), the power of the current study to find genuine effects is likely to be high. The fact that only small effects were found in our sample suggests that the association between maternal psychological distress and cortisol in this type of sample is weakly at best, and probably too complex to be adequately captured with the used techniques. More research is necessary to unravel the underlying mechanisms of this relation and the search for complementary systems (e.g., placental 11ßHSD2, the immune system, catecholamines, microbiome, (epi) genetics, and altered health behaviors) should be continued and extended.

\section{Conflict of interest}

The authors report no biomedical financial interests or potential conflicts of interest.

\section{Acknowledgements}

The authors are grateful to the parents and children for their participation in our study and to the students who helped with the data collection. The PELS study is supported by the national funding agencies of the European Science Foundation (EuroSTRESS - PELS; http:// archives.esf.org/coordinating-research/eurocores/programmes/ eurostress/projects/pels.html) participating in the Eurocores Program EuroSTRESS programme, i.e., the Brain and Cognition Programme of the Netherlands Organisation for Scientific Research (NWO) for the Netherlands. BVdB is project leader of the PELS study; this study is conducted in collaboration with VG and SC. BVdB is supported by European Commission Seventh Framework Programme (FP7-HEALTH. 2011.2.2.2-2 BRAINAGE, grant agreement no: 279281). SC is a Senior Clinical Researcher of the Fund for Scientific Research (FWO) Flanders.

\section{Appendix A. Supplementary data}

Supplementary material related to this article can be found, in the online version, at doi:https://doi.org/10.1016/j.psyneuen.2018.06. 005. 


\section{References}

Arrindel, W.A., Ettema, J.H.M., 1981. Dimensionele structuur, betrouwbaarheid en validiteit van de Nederlandse bewerking van de Symptom Checklist (SCL-90). Swets \& Zeitlinger, Lisse.

Arrindell, W.A., Ettema, J.H.M., 2003. SCL-90. Symptom Checklist. Handleiding bij een multidimensionele psychopathologie-indicator. Swets Test Publishers, Lisse.

Backhaus, J., Junghanns, K., Hohagen, F., 2004. Sleep disturbances are correlated with decreased morning awakening salivary cortisol. Psychoneuroendocrinology 29, $1184-1191$.

Baibazarova, E., van de Beek, C., Cohen-Kettenis, P.T., Buitelaar, J., Shelton, K.H., van Goozen, S.H., 2013. Influence of prenatal maternal stress, maternal plasma cortiso and cortisol in the amniotic fluid on birth outcomes and child temperament at 3 months. Psychoneuroendocrinology 38, 907-915.

Bech, P., 2011. Measuring states of anxiety with clinician-ratedand patient-rated scales. In: Selek, S. (Ed.), Different Views of Anxiety Disorders. InTech, Croatia.

Beijers, R., Buitelaar, J.K., de Weerth, C., 2014. Mechanisms underlying the effects of prenatal psychosocial stress on child outcomes: beyond the HPA axis. Eur. Child Adolec. Psychiatry 23, 943-956.

Bergink, V., Kooistra, L., Lambregtse-van den Berg, M.P., Wijnen, H., Bunevicius, R., van Baar, A., Pop, V., 2011. Validation of the Edinburgh depression scale during pregnancy. J. Psychosom. Res. 70, 385-389.

Bleker, L.S., Roseboom, T.J., Vrijkotte, T.G., Reynolds, R.M., de Rooij, S.R., 2017. Determinants of cortisol during pregnancy - the ABCD cohort. Psychoneuroendocrinology 83, 172-181.

Chida, Y., Steptoe, A., 2009. Cortisol awakening response and psychosocial factors: a systematic review and meta-analysis. Biol. Psychol. 80, 265-278.

Cox, J.L., Holden, J.M., Sagovsky, R., 1987. Detection of postnatal depression. Development of the 10-item Edinburgh postnatal depression scale. Br. J. Psychiatry 150, 782 .

Davis, E.P., Glynn, L.M., Schetter, C.D., Hobel, C., Chicz-Demet, A., Sandman, C.A., 2007 Prenatal exposure to maternal depression and cortisol influences infant temperament. J. Am. Acad. Child Adolesc. Psychiatry 46, 737-746.

de Weerth, C., Buitelaar, J.K., 2005. Physiological stress reactivity in human pregnancy-a review. Neurosci. Biobehav. Rev. 29, 295-312.

Edwards, S., Clow, A., Evans, P., Hucklebridge, F., 2001. Exploration of the awakening cortisol response in relation to diurnal cortisol secretory activity. Life Sci. 68, 2093-2103.

Entringer, S., Buss, C., Shirtcliff, E.A., Cammack, A.L., Yim, I.S., Chicz-DeMet, A., Sandman, C.A., Wadhwa, P.D., 2010. Attenuation of maternal psychophysiological stress responses and the maternal cortisol awakening response over the course of human pregnancy. Stress 13, 258-268.

Evans, L., Myers, M., Monk, C., 2008. Pregnant women's cortisol is elevated with anxiety and depression - but only when comorbid. Arch. Women's Ment. Health 11, 239-248.

Giesbrecht, G.F., Campbell, T., Letourneau, N., Kooistra, L., Kaplan, B., 2012. Psychological distress and salivary cortisol covary within persons during pregnancy. Psychoneuroendocrinology 37, 270-279.

Giesbrecht, G.F., Campbell, T., Letourneau, N., Kaplan, B.J., 2013. Advancing gestation does not attenuate biobehavioural coherence between psychological distress and cortisol. Biol. Psychol. 93, 45-51.

Glover, V., 2014. Maternal depression, anxiety and stress during pregnancy and child outcome; What needs to be done. Best Practice Res. Clin. Obst. Gynaecol. 28, 25-35.

Goldstein, H., 2003. Multilevel Statistical Models. Wiley, New York.

Harville, E.W., Savitz, D.A., Dole, N., Herring, A.H., Thorp, J.M., Light, K.C., 2007 Patterns of salivary cortisol secretion in pregnancy and implications for assessment protocols. Biol. Psychol. 74, 85-91.

Heck, R.H., Thomas, S.L., Tabata, L.N., 2010. Multilevel and Longitudinal Modeling With IBM SPSS. Routledge, New York.

Hellgren, C., Åkerud, H., Skalkidou, A., Sundström-Poromaa, I., 2013. Cortisol awakening response in late pregnancy in women with previous or ongoing depression. Psychoneuroendocrinology 38, 3150-3154.

Himes, K.P., Simhan, H.N., 2011. Plasma corticotropin-releasing hormone and cortisol concentrations and perceived stress among pregnant women with preterm and term birth. Am. J. Perinatol. 28, 443-448.

Hompes, T., Vrieze, E., Fieuws, S., Simons, A., Jaspers, L., Van Bussel, J., Schops, G., Gellens, E., Van Bree, R., Verhaeghe, J., Spitz, B., Demyttenaere, K., Allegaert, K., Van den Bergh, B., Claes, S., 2012. The influence of maternal cortisol and emotional state during pregnancy on fetal intrauterine growth. Psychiatry Res. 72, 305-315.

Hruschka, D.J., Kohrt, B.A., Worthman, C.M., 2005. Estimating between- and withinindividual variation in cortisol levels using multilevel models. Psychoneuroendocrinology 30, 698-714.

Huizink, A.C., Mulder, E.J.H., Robles de Medina, P.G., Visser, G.H.A., Buitelaar, J.K., 2004. Is Pregnancy anxiety a distinctive syndrome? Early Hum. Dev. 79, 81-91.

Kalra, S., Einarson, A., Karaskov, T., Van Uum, S., Koren, G., 2007. The relationship between stress and hair cortisol in healthy pregnant women. Clin. Invest. Med. 30, E103-107.

Kane, H.S., Dunkel Schetter, C., Glynn, L.M., Hobel, C.J., Sandman, C.A., 2014. Pregnancy anxiety and prenatal cortisol trajectories. Biol. Psychol. 100, 13-19.

Kivlighan, K.T., DiPietro, J.A., Costigan, K.A., Laudenslager, M.L., 2008. Diurnal rhythm of cortisol during late pregnancy: associations with maternal psychological wellbeing and fetal growth. Psychoneuroendocrinology 33, 1225-1235.

Koelewijn, J.M., Sluijs, A.M., Vrijkotte, T.G.M., 2017. Possible relationship between general and pregnancy-related anxiety during the first half of pregnancy and the birth process: a prospective cohort study. BMJ Open 7, e013413.
Kupper, N., de Geus, E.J., van den Berg, M., Kirschbaum, C., Boomsma, D.I., Willemsen, G., 2005. Familial influences on basal salivary cortisol in an adult population. Psychoneuroendocrinology 30, 857-868.

Lahti, M., Savolainen, K., Tuovinen, S., Pesonen, A.-K., Lahti, J., Heinonen, K., Hämäläinen, E., Laivuori, H., Villa, P.M., Reynolds, R.M., Kajantie, E., Räikkönen, K., 2017. Maternal depressive symptoms during and after pregnancy and psychiatric problems in children. J. Am. Acad. Child. Adolesc. Psychiatry 56, 30-39 e37.

Menard, S., 1995. Applied Logistic Regression Analysis. Sage, Thousand Oaks, CA.

Myers, R., 1990. Classical and Modern Regression with Applications, 2nd ed. Duxbury, Boston, MA.

O'Connor, T.G., Heron, J., Golding, J., Beveridge, M., Glover, V., 2002. Maternal antenatal anxiety and children's behavioural/emotional problems at 4 years. Report from the Avon longitudinal study of parents and children. Br. J. Psychiatry: J. Ment. Sci. $180,502-508$.

O'Connor, T.G., Tang, W., Gilchrist, M.A., Moynihan, J.A., Pressman, E.K., Blackmore, E.R., 2014. Diurnal cortisol patterns and psychiatric symptoms in pregnancy: shortterm longitudinal study. Biol. Psychol. 96, 35-41.

Obel, C., Hedegaard, M., Henriksen, T.B., Secher, N.J., Olsen, J., Levine, S., 2005. Stress and salivary cortisol during pregnancy. Psychoneuroendocrinology 30, 647-656.

Petraglia, F., Hatch, M.C., Lapinski, R., Stomati, M., Reis, F.M., Cobellis, L., Berkowitz, G.S., 2001. Lack of effect of psychosocial stress on maternal corticotropin-releasing factor and catecholamine levels at 28 weeks' gestation. J. Soc. Gynecol. Invest. 8, 83-88.

Räikkönen, K., Seckl, J.R., Pesonen, A.K., Simons, A., Van den Bergh, B.R., 2011. Stress, glucocorticoids and liquorice in human pregnancy: programmers of the offspring brain. Stress 14, 590-603.

Raudenbush, S.W., Bryk, A.S., 2002. Hierarchical Linear Models, 2nd ed. Sage, Thousand Oaks, CA.

Salacz, P., Csukly, G., Haller, J., Valent, S., 2012. Association between subjective feelings of distress, plasma cortisol, anxiety, and depression in pregnant women. Eur. J. Obstet. Gynecol. Reprod. Biol. 1, 1.

Sandman, C.A., Glynn, L., Schetter, C.D., Wadhwa, P., Garite, T., Chicz-DeMet, A., Hobel, C., 2006a. Elevated maternal cortisol early in pregnancy predicts third trimester levels of placental corticotropin releasing hormone $(\mathrm{CRH})$ : priming the placental clock. Peptides 27, 1457-1463.

Sandman, C.A., Glynn, L., Schetter, C.D., Wadhwa, P., Garite, T., Chicz-DeMet, A., Hobel, C., 2006b. Elevated maternal cortisol early in pregnancy predicts third trimester levels of placental corticotropin releasing hormone (CRH): priming the placental clock. Peptides 27, 1457-1463.

Shea, A.K., Streiner, D.L., Fleming, A., Kamath, M.V., Broad, K., Steiner, M., 2007a. The effect of depression, anxiety and early life trauma on the cortisol awakening response during pregnancy: preliminary results. Psychoneuroendocrinology 32, 1013-1020.

Shea, A.K., Streiner, D.L., Fleming, A., Kamath, M.V., Broad, K., Steiner, M., 2007b. The effect of depression, anxiety and early life trauma on the cortisol awakening response during pregnancy: preliminary results. Psychoneuroendocrinology 32, 1013-1020.

Simon, C.D., Adam, E.K., Holl, J.L., Wolfe, K.A., Grobman, W.A., Borders, A.E.B., 2016. Prenatal stress and the cortisol awakening response in African-American and caucasian women in the third trimester of pregnancy. Mater. Child. Health J. 20, 2142-2149.

Snijders, T.A.B., Bosker, R., 2011. Multilevel Analysis: An Introduction to Basic and Advanced Multilevel Modeling. Sage Publications Ltd.

Stalder, T., Kirschbaum, C., Kudielka, B.M., Adam, E.K., Pruessner, J.C., Wüst, S., Dockray, S., Smyth, N., Evans, P., Hellhammer, D.H., Miller, R., Wetherell, M.A., Lupien, S.J., Clow, A., 2016. Assessment of the cortisol awakening response: expert consensus guidelines. Psychoneuroendocrinology 63, 414-432.

Suglia, S.F., Staudenmayer, J., Cohen, S., Enlow, M.B., Rich-Edwards, J.W., Wright, R.J., 2010. Cumulative stress and cortisol disruption among Black and Hispanic pregnant women in an urban cohort. Psychol. Trauma. 2, 326-334.

Valsamakis, G., Papatheodorou, D.C., Chalarakis, N., Vrachnis, N., Sidiropoulou, E.J., Manolikaki, M., Mantzou, A., Margeli, A., Papassotiriou, I., Chrousos, G.P., Mastorakos, G., 2017. In pregnancy increased maternal STAI trait stress score shows decreased insulin sensitivity and increased stress hormones. Psychoneuroendocrinology 84, 11-16.

Van den Bergh, B.R.H., 1990. The influence of maternal emotions during pregnancy on fetal and neonatal behavior. J. Perinatal. Prenat. Psychol. Health 5, 119-130.

Van den Bergh, B.R.H., Marcoen, A., 2004. High antenatal maternal anxiety is related to ADHD symptoms, externalizing problems, and anxiety in 8- and 9-year-olds. Child Dev. 75, 1085-1097.

Van den Bergh, B.R.H., Van Calster, B., Pinna Puissant, S., Van Huffel, S., 2008. Selfreported symptoms of depressed mood, trait anxiety and aggressive behavior in postpubertal adolescents: associations with diurnal cortisol profiles. Horm. Behav. 54, 253-257.

Van den Bergh, B.R.H., van den Heuvel, M.I., Lahti, M., Braeken, M., de Rooij, S.R., Entringer, S., Hoyer, D., Roseboom, T., Räikkönen, K., King, S., Schwab, M., 2017. Prenatal developmental origins of behavior and mental health: the influence of maternal stress in pregnancy. Neurosci. Biobehav. Rev. http://dx.doi.org/10.1016/j. neubiorev.2017.07.003. Epub ahead of print.

van den Heuvel, M.I., Johannes, M.A., Henrichs, J., Van den Bergh, B.R.H., 2015 Maternal mindfulness during pregnancy and infant socio-emotional development and temperament: the mediating role of maternal anxiety. Early Hum. Dev. 91, 103-108.

Van der Ploeg, H.M., Defares, P.B., Speilberger, C.D., 2000. Handleiding bij de Zelfbeoordelings Vragenlijst ZBV: Een nederlandstalige bewerking van de SpieIlberger State-Trait Anxiety Inventory, STAI-DY, 2nd ed. Swets \& Zeitlinger, Lisse.

Voegtline, K., Costigan, K., Kivlighan, K., Laudenslager, M., Henderson, J., DiPietro, J., 2013. Concurrent levels of maternal salivary cortisol are unrelated to self-reported psychological measures in low-risk pregnant women. Arch. Women's Ment. Health 
16, 101-108.

Walder, D.J., Laplante, D.P., Sousa-Pires, A., Veru, F., Brunet, A., King, S., 2014. Prenatal maternal stress predicts autism traits in $6 \frac{1}{2}$ year-old children: project ice storm. Psychiatry Res. 219, 353-360.

Zijlmans, M.A., Riksen-Walraven, J.M., de Weerth, C., 2015. Associations between maternal prenatal cortisol concentrations and child outcomes: a systematic review. Neurosci. Biobehav. Rev. 53, 1-24.

Zijlmans, M.A.C., Beijers, R., Riksen-Walraven, M.J., de Weerth, C., 2017. Maternal late pregnancy anxiety and stress is associated with children's health: a longitudinal study. Stress 1-10. 